1997 Chasing the Blood Tie: Surrogate Mothers, Adoptive Mothers, and Fathers. In Situated Lives: Gender and Culture in Everyday Life, edited by Louise Lamphere, Helena Ragoné, and Patricia Zavella. New York: Routledge.

SCHNEIDER, DAVID M.

1995 Schneider on Schneider: The Conversion of the Jews and Other Anthropological Stories by David M. Schneider as told to Richard Handler. Durham: Duke University Press.

\title{
On Sousa's Epidemiological Approach
}

\author{
Christophe Heintz*
}

My comments bear on Sousa's claim to provide an epidemiological analysis of a scientific representation. I will argue that Sousa is particularly justified to call on Sperber's epidemiology of representation for the study of the history of science, but I will question the extent to which Sousa actually applies the epidemiology of representations to the fall of kinship studies in anthropology. An epidemiological study requires not only the acceptance of some theoretical assumptions, but also the exploitation of the analogy with medical epidemiology and the exploitation of the interface that is made apparent between social anthropology and cognitive psychology.

\section{The Epidemiology of Scientific Representations}

Since Latour and Woolgar's "Laboratory Life" (1986), there has been a growing interest in anthropological studies of science. Yet, these anthropological approaches remain highly controversial. This is partly due to their

*Institut Jean Nicod, EHESS. E-mail: Christophe.Heintz@ehess.fr. 
radical naturalistic stance, which forces them to put a ban on non-natural entities like Truth, Reason or Progress, which are the common irreducible explanandum of traditional accounts of scientific events. ${ }^{1}$ This ban should be even more accentuated in an epidemiological analysis, which has for motto "Whatever has causal powers has them in virtue of its material properties." 2 Sousa's account of the fall of kinship meets this requirement. Sousa points out that "a common explanatory picture in anthropology is that the decline of kinship as an object of study is just a result of the progress of knowledge" (my italics). His explanation of the decline of kinship contrasts with teleological accounts and appeals to the causal action of some other representations, viz. the hermeneutic view of anthropology and the association of kinship studies with the attempt to transform anthropology into a natural science. When claiming to apply the epidemiology of representations to the explanation of a scientific event, Sousa poses himself as a cognitive anthropologist of science. This field of study is, I believe, the most promising approach for a naturalistic study of science. ${ }^{3}$

Also, the epidemiology of representations seems well suited to the history of scientific ideas. First, the epidemiological framework allows for a dynamic analysis of cultural phenomena: Time is an essential component of the epidemiological framework because cultural phenomena are characterized as "long and lasting" social cognitive causal chains (Sperber, 2001). Cultural phenomena imply the stabilization of some representations, i.e., their remaining recognizably similar to antecedent representations across time. Now, as Sousa points out, epidemiological studies have focused on the conditions, and in particular the cognitive conditions, which allow for the resilience and the continuity of content of some representations. This focus, I want to add, is justified because most social events do not lead to stable representations. Therefore, something more is needed

${ }^{1}$ Giere (1999) argues that the Enlightenment view of science is responsible for most of the science war, and in particular for the reactions against 'science studies.' He then observe that holding a different view of science (e.g. science as an object for empirical study) does not necessarily goes against any scientific enterprise.

${ }^{2}$ Truth is a semantic notion, so it has no causal power as such. Appeal to Reason and Progress need at least much further analysis than usually done in traditional account, in order to provide a description of the causal mechanisms refereed to.

${ }^{3}$ Arguments and illustrations of this otherwise bold assertion shall be found in the June 2004 issue of this journal, which will be consecrated to Cognitive Anthropology of Science. 
to make a cultural representation, and it is this something more that is important for explaining culture. But of course, Sousa is right to assert that cultural change also falls into the epidemiological rationale: the stability of a cultural representation is always partial and depends on the environmental, contingent, conditions that sustained it. Studying how changes in the environmental conditions affect the stability of a representation is indeed a genuine epidemiological question, i.e., one that can fruitfully be answered within this theoretical framework.

Second, the epidemiology of representation, with its stress on individual cognition as constitutive of cultural phenomena, suits well our intuitions of what happens in science: scientists think, observe, test, etc. they produce their own mental representations. Then, they communicate their results: they produce scientific papers, talks, lectures, etc., i.e. they produce public representations.

Third: as we all know, most representations produced by scientists do not stabilize. The central question of the history of science can therefore be rephrased in epidemiological terms: what are the processes of distribution of representations among the scientific community that allow such and such representations to stabilize and such and such other representations to cease to be important and widespread?

\section{Applying the Epidemiological Framework}

Sousa's paper has the subtitle "Towards an epidemiological explanation." Sousa does not therefore pretend to give a full epidemiological explanation, but rather the first analysis for it. I think Sousa is right to be both ambitious and modest in his claim to apply the epidemiology of representations. The rightness of the ambition has been shown above. Through the entire article, Sousa shows his commitment with the theoretical assumptions of the epidemiology of representations. He talks about mental and public representations, about the distribution of such representations, and he regularly asserts that his anthropological approach is a search for the causes of the fall of kinship. Obviously, Sousa has chosen his camp: he does not stand with the hermeneutic, postmodernist credo. On the contrary, he can be situated on the right hand side of the axis of his schema "Whither anthropology?" ... as any epidemiologist would be expected to be. 
The epidemiology of representations, however, is more than a set of epistemological assertions. It is a theoretical framework that provides some specific means of analysis. Two theoretical tools are straightforwardly at hand to the epidemiologist: first, the analogy with medical epidemiology and second the interface with cognitive psychology. My frustration is that these theoretical tools are not fully exploited by Sousa, so I will try to briefly point at some possible uses of those tools for the anthropological analysis of the fall of kinship.

Medical epidemiology: the epidemiology of representations takes the concept of distribution from medical epidemiology. But while Sousa characterizes the fall of kinship in terms of distribution of representations in the community of social anthropologists, he does not provide a thorough investigation. One difficulty with the epidemiology of representations is to pin up the representation whose distribution is to be studied. This is a difficulty because, as Sperber emphasizes, there is no genuine reproduction of representations. Consequently, representations are always altered when going through cognitive processes and social interactions. So what is Sousa referring to when he talks about the fall of kinship? Concepts in the human sciences are cases in point for the transformation of representations: every author has a different understanding of the concept! As Sousa shows, this holds for kinship. And yet, we still have something that is "recognizably similar." I suggest that the most easily recognizable and relevant representation is the lexical item "kinship". On the one hand, this representation is relevant because one can easily assume that when anthropologists talk about kinship, they intend to talk about the same thing (i.e. there are no obvious cases of synonymy). On the other hand, the lexical item is easily traceable and the historian can benefit from the fact that scientific public representations are largely written representations. "Kinship" appears in books, articles and title of courses, which can be dated and whose importance, in terms of distribution, can be measured. With the lexeme "kinship" as the immediate target of empirical investigation, the epidemiology of representations can, as medical epidemiology, take benefit of statistical data. How many course books with kinship in their title have been sold in 1965? Did the number continuously decrease up to now? Has the term "kinship" moved to chapters of course books or has it totally disappeared from teaching? What about the geographical distribution? Is kinship more used in British 
Anthropology than in American Anthropology? Another advantage with scientific representations, apart from their traceability, is that the community where the distribution is to be studied is rather clearly bounded. Thus the historian can focus on specific journals, departments, and book series. Sousa clearly focuses on the fall of kinship in anthropology. But the dynamics of scientific disciplines are never wholly internal and it can be fruitful to consider some of the external influence. For instance, kinship terms can surely be found in the current problems of bioethics. With regard to the later, one can surely perceive a demand for kinship representations and one may wonder if the fall of kinship in anthropology is not attenuated by changes in the means of human reproduction (see e.g. Strathern, 1992; Edwards, 2000).

An epidemiological analysis of the term "kinship" in anthropology and in influential domains, enriched with statistical data, could provide a rigorous meaning to "the fall of kinship." Without such data, the reader is left with his own intuition in order to complete Sousa's brief characterization: "the idea of kinship became and remained an unsound theoretical concept in the minds of many anthropologists, with the consequent decrease of public productions such as lectures, colloquia, articles and books about kinship." Although the intuition with regard to the fall of kinship may be pretty clear among anthropologists who can rely on their own experience, other readers may have no ideas, or misleading ideas, on the topic.

The social anthropology/cognitive psychology interface: the epidemiology of representations makes this interface apparent by showing the importance of individual cognitive processes in the causal chains that constitute cultural phenomena. What are the causal relations between scientific cognition and the fall of kinship? Sousa's work bears on an important phenomenon in science, viz. the relation between a representation and its validating context. Most scientific representations are not accepted at face value, they are not the direct produce of some cognitive process. Rather, scientific representations are historically elaborated through social interactions and depend, for their acceptation, on other complex representations. ${ }^{4}$ Yet, the fact that

\footnotetext{
${ }^{4}$ More precisely and using Sperber's terminology and analysis, the fact that a scientific representation is taken to be true by an individual depends on the meta-representational context in which the representation is embedded. For instance, the fact that I believe
} 
scientific discourse is only remotely connected to intuitions (mere produce of cognitive processes) does not render cognitive processes irrelevant for the study of Science. In fact, the causal relations between cognitive processes and cultural production such as scientific representations have been worked out by Sperber (1996), who appeals to the notion of "cognitive attractor." The basic idea is that individual's production of representations is biased or constrained by human cognitive abilities in such a way that representations tend to stabilize around attractors. This is because our cognitive apparatus allows us to reproduce more faithfully only a limited set of representations.

In Sousa's case, it is hard to see which cognitive ability or direct intuition led to the fall of kinship. On the contrary, Sousa argues that this historical event is due to high level representations: the meta-representations that are epistemological norms. Yet, cognitive attractors are not only determined by human innate cognitive apparatus, mutual cognitive environments can constraint culture production so as to constitute cognitive attractors. A mutual cognitive environment is determined by the set of representations that can easily be retrieved either form memory or from the environment by a given community of people (Sperber \& Wilson 1986). Because mutual cognitive environment determine scientists' thought, they also determine scientific knowledge production. Kuhnian paradigms in the history of science constitute mutual cognitive environments, but the notion of cognitive environment is suppler than the notion of paradigm and can characterize non-revolutionary changes in the history of science. So, whether the hermeneutic turn in anthropology is a Kuhnian revolution or not, it can certainly qualify as a change in the mutual cognitive environment. Also, the fact that mutual cognitive environments can generate cognitive attractors is shown by the numerous cases where a given discovery is independently made by different scientists partaking the same cognitive environment (e.g. because they live at the same time, in a similar scientific environment).

The notion of cognitive attractor, however, cannot account for the fall of a concept. Fortunately, Sousa's clever twist of the epidemiological

that atoms exist depends on my holding representations such as 'my professor told me that atoms exist,' and, for physicists, on other more complex representations involving the notion of atom. Atoms can never be directly perceived. In short, scientific representations are reflective representations, as opposed to intuitive representations (see Sperber 1997). 
framework for the study of cultural change leads to the notion of cognitive repulsor. The mutual cognitive environment constituted by the epistemology of hermeneutics created a cognitive repulsor for kinship studies. If Sousa's analysis can be rephrased in these terms, then it could be enriched by an account of the mode of action of the cognitive repulsor. From Sousa's parsimonious assertions one can conclude that it did not act because of theoretical incompatibility between hermeneutic epistemology and kinship theories. Rather, Sousa suggests that the cognitive repulsor acted by depriving kinship studies from motivational force: because kinship studies went in the wrong direction, the project itself was assumed to be useless, i.e. not worth the effort.

What all this jargon for? The terms "cognitive attractor," "cognitive repulsor" and "cognitive environment" allow a rephrasing of Sousa's analysis that should enable further investigations of the cognitive processes at work in the fall of kinship. Without further investigations of the cognitive causes of the cultural phenomenon, the analysis risks to remain at the ideological level, studying the influence of one set of ideas over another set of ideas.

\section{REFERENCES}

EDWARDS, J.

2000 Born and bred: idioms of kinship and new reproductive technologies in England. Oxford: OUP.

GiERE, R.

1999 Science without Laws. Chicago: University of Chicago Press.

LATOUR, B. \& WoOlgar, S.

1986 Laboratory Life. Princeton: Princeton University Press.

Sperber, D. \& Wilson, D.

1986 Relevance: Communication and Cognition. Oxford: Blackwell.

SPERBER, D.

1996 Explaining culture: A naturalistic approach. Oxford: Blackwell.

1997 'Intuitive and Reflective Beliefs.' Mind and Language 12(1): 67-83.

2001 Conceptual tools for a natural science of society and culture (Radcliffe-Brown

Lecture in Social Anthopology 1999). In Proceedings of the British Academy 111: 297-317.

STRATHERN, M.

1992 Reproducing the future: essays on anthropology, kinship and the new reproductive technologies. Manchester: Manchester University Press. 